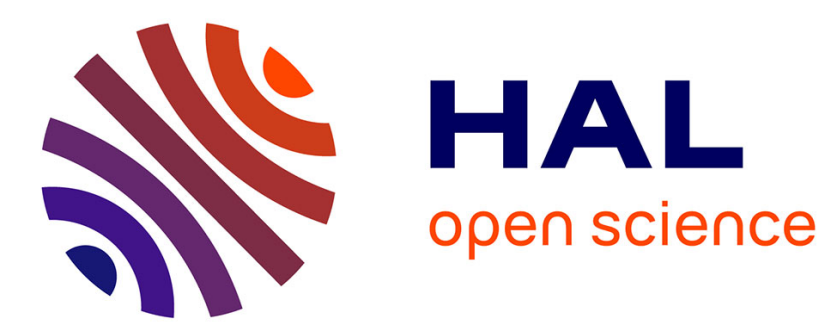

\title{
PECULIAR HOT ELECTRON EFFECTS IN CROSSED ELECTRIC AND MAGNETIC FIELDS \\ T. Kurosawa
}

\section{To cite this version:}

T. Kurosawa. PECULIAR HOT ELECTRON EFFECTS IN CROSSED ELECTRIC AND MAGNETIC FIELDS. Journal de Physique Colloques, 1981, 42 (C7), pp.C7-377-C7-386. 10.1051/jphyscol:1981746 . jpa-00221683

\section{HAL Id: jpa-00221683 https://hal.science/jpa-00221683}

Submitted on 1 Jan 1981

HAL is a multi-disciplinary open access archive for the deposit and dissemination of scientific research documents, whether they are published or not. The documents may come from teaching and research institutions in France or abroad, or from public or private research centers.
L'archive ouverte pluridisciplinaire HAL, est destinée au dépôt et à la diffusion de documents scientifiques de niveau recherche, publiés ou non, émanant des établissements d'enseignement et de recherche français ou étrangers, des laboratoires publics ou privés. 
PECULIAR HOT ELECTRON EFFECTS IN CROSSED ELECTRIC AND MAGNETIC FIELDS

T. Kurosawa

Department of Physics, Chwo University, Tokyo, Japan

\begin{abstract}
Résumé. - Ies effets de porteurs chauds qui sont attendus (ou quelquefois observés) dans les champs croisés électrique (aussi statiques que de micro-onde) et magnetique (pas quantique) ont été étudiés. Ce qui nous intéresse le plus est le cas où l'interaction entre le porteur et le phonon optique est plus forte que les autres processus de diffusion. Dans les champs électriques statiques l'effet principal est l'accumulation de porteurs dans la région spéciale de l'espace de la quantité de mouvement qui produit, par exemple, I'inversion de population, la conductivité différentielle négative, la supraradiance et le déplacement caractéristique de résonance du cyclotron du germanium de type-p. Dans le champ intense de micro-onde, remarquables sont les caractères systêmatiques qui se montrent dans le mouvement de porteur et aonnent quelques effets particuliers comme le "bunching" dans I'espace de quantité de mouvement.
\end{abstract}

Abstract. - Hot carrier effects expected (in some cases observed) in crossed electric (both static and microwave) and magnetic (not quantum) fields are surveyed. The case that the carrieroptical phonon interaction is strong compared with the other scattering processes is of main concern. In static electric fields, the principal effect is the carrier accumulation in a special region of the momentum space which leads to, e.g., population inversion, negative differential conductivity, superradiance and specific shift of cyclotron resonance in p-type germanium. In intense microwave fields, remarkable is systematic characters exhibited by the carrier motion which bring about some peculiar effects such as the bunching in the momentum space.

\title{
\$1. Introduction
}

One of the interests in the study of physics on hot carriers in semiconductors is the possibility of finding peculiar phenomena, which reflect the characteristic features of the scattering processes, the motion, and the distribution function of the carrier. A good hunting field for such phenomena can be found with hot carriers in the situation that the carrier-optical phonon interaction is strong compared with the other scattering processes and the thermal energy $\mathrm{kT}$ is much smaller than the optical phonon energy $\hbar \omega_{o p}$ : The collision time of the carrier is very long when the energy $\varepsilon$ is smaller than thop' and the carrier is accelerated rather freely until it arrives 
at the optical phonon energy, then rapidly emits the optical phonon. The distribution function is quite different from that of a simple hot carrier concept with a definite electron temperature and leads to some strange effects.

The application of magnetic field makes the phenomena more fruitful. Several peculiar hot carrier effects expected or observed in this case are surveyed; in $\$ 2$ the effects in static electric fields, and in $\$ 3$ those in intense microwave fields.

\section{\$2. Static Electric Fields}

Figure 1 shows a typical example of the peculiar hot carrier distribution function on the $\mathrm{p}_{z}=0$ plane in the momentum space obtained from a Monte Carlo calculation under the crossed electric and magnetic fields of appropriate strengths: The free acceleration of a carrier in the $\mathrm{p}$-space is represented by a circular motion with the cyclotron frequency $\omega_{C}\left(=\mathrm{eH}_{z} / \mathrm{m}^{*} \mathrm{C}\right)$ around an axis denoted by $\mathrm{C}$ in the figure where $p_{x}=0$ and $p_{y}=p_{C} \equiv m^{*} C E_{x} / H_{z}$ The carrier accumulates in a circular region around $C$ where the energy is always smaller than the optical phonon energy, while the

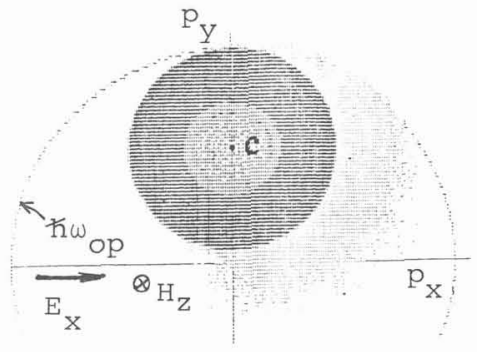

Fig.1. circular motion is interrupted very rapidly by the optical phonon emission when the carrier is outside of the region [1].

A detectable effect of the accumulation is a larger Hall angle than that expected from the estimation neglecting it. This has been observed in. AgCl and AgBr by Komiyama, Masumi and Kajita [2] and in p-type germanium by Komiyama and Spies [3]. Another evidence for the accumulation which seems more conclusive has been obtained also by Komiyama et al [2]: Figure 2 shows the current response $J_{z}$ to a weak electric field additionally applied along the magnetic field, plotted against $\mathrm{H}_{\mathrm{z}}$ for some fixed values of $E_{x}$. Physically, the ordinate is proportional to the effective collision time (namely, the average duration of the free motion) of the carrier, and the dotted lines represent those expected from the assumption that the carrier repeats the streaming motion along the trajectory $O P$ as shown in the inset. The vertical arrows indicate the

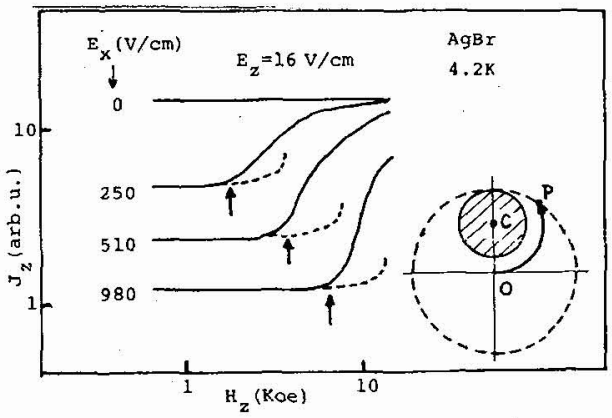

Fig.2. An experimental evidence for the carrier accumulation (see text). 
magnetic field where the energy of the point $\mathrm{C}\left(=\mathrm{m}^{*}\left(\mathrm{CE} \mathrm{x}_{\mathrm{z}} / \mathrm{H}_{\mathrm{z}}\right)^{2} / 2\right)$ is equal to fiw op. As $\mathrm{H}_{\mathrm{z}}$ increases above this value (i.e., the point $\mathrm{C}$ enters the circle of $\varepsilon=\hbar \omega_{o p}$ ', the current $J_{z}$ becomes much larger than the value of the dotted lines. This means evidently that the carrier spends the considerable fraction of the time in the accumulation region.

Degree of Accumulation

To estimate the degree of the accumulation, we adopt a simple rate equation approach. We divide the momentum space into three regions: (1) The region where the accumulation takes place, (2) the region outside of the accumulation region and of $\varepsilon<\hbar \omega_{o p}$, and (3) the region of $\varepsilon>\hbar \omega_{o p}$. If we denote the number of carriers in these regions by $\mathrm{n}_{1}, \mathrm{n}_{2}$ and $\mathrm{n}_{3}$, we may write the following approximate equations:

$$
\begin{aligned}
& \frac{d n_{1}}{d t}=w_{o p} p_{k} n_{3}-\frac{n_{1}}{\tau} \\
& \frac{d n_{2}}{d t}=w_{o p}\left(1-P_{k}\right) n_{3}+\frac{n_{1}}{\tau}-\frac{\omega_{c}}{\phi_{2}} n_{2} \\
& \frac{d n_{3}}{d t}=\frac{w_{c}}{\phi_{2}} n_{2}-w_{o p} n_{3},
\end{aligned}
$$

where $W_{o p}$ is the optical phonon emitting rate, $\tau$ the average collision time in the region ( 1 , , $\phi_{2}$ the average rotating angle in the region (2), and $P_{k}$ is the probability that the carrier is scattered into the region (1) after the optical phonon emission.

In the steady state, we have

$$
\mathrm{n}_{3} / \mathrm{n}_{2}=\omega_{\mathrm{C}} / \mathrm{w}_{\mathrm{op}} \phi_{2} \ll 1
$$

and

$$
\mathrm{n}_{1} / \mathrm{n}_{2}=\omega_{\mathrm{c}} \tau \mathrm{P}_{\mathrm{k}} / \phi_{2}
$$

Thus, the latter expression gives the degree of the accumulation. Here, $\phi_{2}$ is usually $2 \sim 3$, while $\omega_{c}{ }^{\tau}$ strongly depends on the experimental conditions but may be estimated rather easily in each case. As for $\mathrm{P}_{\mathrm{k}}$, there is no simple way to estimate $i t$, but the results of numerical calculations can be represented well by using only two parameters when $\omega_{c} \tau>1$.

The values of $\mathrm{P}_{\mathrm{k}}$ are plotted in Fig. 3 for polar optical scattering and in Fig. 4 for nonpolar scattering, against two parameters $\mathrm{B}_{\mathrm{op}} / \omega_{\mathrm{C}}$ and $\mathrm{p}_{\mathrm{C}} / \mathrm{p}_{\mathrm{op}}$. The parameter $\mathrm{p}_{\mathrm{C}} / \mathrm{p}_{\mathrm{op}}$ denotes the place of the point $\mathrm{C}$ relative to the momentum at the optical phonon energy $p_{o p}\left(=\sqrt{2 m^{*} \kappa \omega_{o p}}\right)$. on the other hand, $B_{\text {op }}$ is the coefficient for the scattering rate by the optical phonon, which is written as

$$
w_{\text {op }}(\varepsilon)=B_{\text {op }} \sqrt{\varepsilon / K \omega_{\text {op }}-1}
$$

for nonpolar scattering, and 


$$
W_{\text {op }}(\varepsilon)=\frac{{ }_{\text {op }}}{2} \sqrt{\frac{\hbar \omega o p}{\varepsilon}} \log \left(\frac{\sqrt{\varepsilon}+\sqrt{\varepsilon-\hbar \omega o p}}{\sqrt{\varepsilon-\sqrt{\varepsilon-\hbar \omega}}}\right)
$$

for polar scattering, where the occupation number of the optical phonon is practically 0 in the present case and only the spontaneous emission

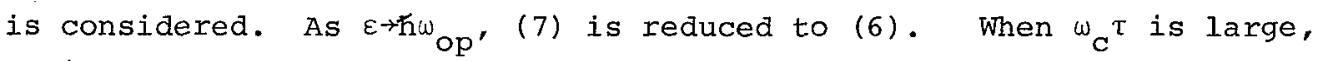
$\mathrm{P}_{\mathrm{k}}$ is nearly independent of the details of scattering processes for $\varepsilon<\hbar \omega_{\text {op }}$.

The following features are remarkable: (I) As $\mathrm{B}_{\mathrm{Op}} / \omega_{\mathrm{C}}$ increases, the residual energy of carriers after the phonon emission decreases and $P_{k}$ becomes small especially for large $p_{c} / p_{o p} \cdot$ (2) Because of the forward scattering nature of the polar scattering, $P_{k}$ is generally 1.503 times larger than that for the nonpolar case.

As a numerical example, if we consider the case that $\mathrm{B}_{\mathrm{op}} / \omega_{\mathrm{C}}=5$ and $\mathrm{p}_{\mathrm{c}} / \mathrm{p}_{\text {op }}=0.65$, we have $\mathrm{P}_{\mathrm{k}}=0.12$ and 0.074 for polar and nonpolar cases respectively. Furthermore if we assume $\omega_{C} \tau=15$ and $\phi_{2}=2, n_{1} / n_{2}$ becomes 0.90 and 0.56 , which are appreciable accumulations indeed when compared with the volume ratio of the two regions in the momentum space, 0.086 . In the case of Fig.1, $\mathrm{p}_{C} / \mathrm{p}_{\mathrm{op}}=0.55, \mathrm{~B}_{\mathrm{op}} / \omega_{\mathrm{C}}=20$ (nonpolar) $\mathrm{P}_{\mathrm{k}} \simeq 0.20, \omega_{\mathrm{c}} \tau \simeq 30, \phi_{2} \simeq 2.1$ and $\mathrm{n}_{1} / \mathrm{n}_{2} \simeq 2.9$.

Negative Differential Conductivity

The accumulation of the carrier and its disappearance with the increase of $\mathrm{E}_{\mathrm{x}}$ lead to negative differential conductivity (NDC) $[1,4]$. Simply this is explained in terms of the decreasing drift velocity due to the carrier transfer from the accumulation region with average velocity $\mathrm{p}_{\mathrm{C}} / \mathrm{m}^{*}$ to the streaming state with about $\mathrm{p}_{\mathrm{op}} / 2 \mathrm{~m}^{*}\left(<\mathrm{p}_{\mathrm{C}} / \mathrm{m}^{*}\right)$ with increasing $E_{x}$.

In the present case, however, the current makes a large angle to the electric field and the NDC must be defined differently from the usual case [5]: The rapid decrease in the Hall angle with $\mathrm{E}_{\mathrm{x}}$ also causes or enhances the NDC. However, we summarize only the results here, since the situation is rather complicated.

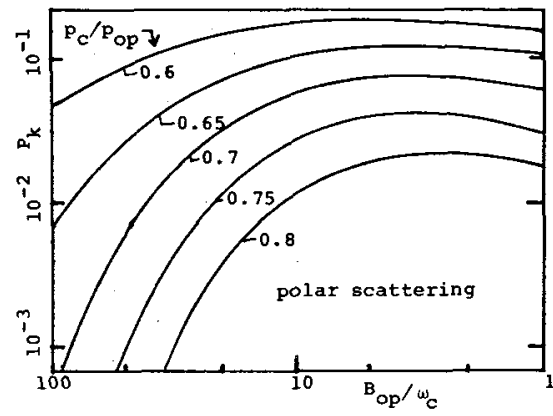

Fig. 3. The probability $\mathrm{P}_{\mathrm{k}}$ (see eq. (1)) for polar case.

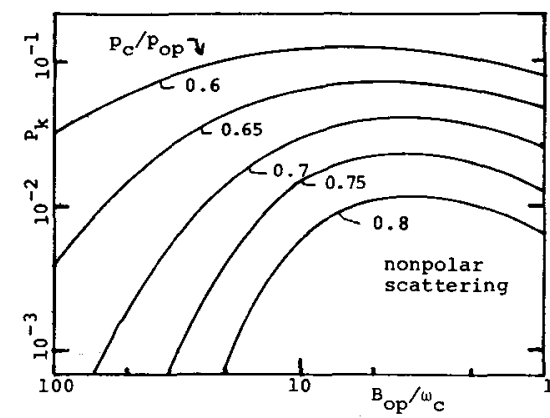

Fig.4. The probability $\mathrm{P}_{\mathrm{k}}$ for nonpolar case. 
There is a lower limit for $\omega_{C}{ }^{T}$ to cause the NDC. The lower limit is $15 \sim 20$ and rather slowly dependent on $\mathrm{B}_{\mathrm{op}} / \omega_{\mathrm{c}}$ in the polar case, while it is generally smaller in the nonpolar case; it is about 10 at $B_{o p} / \omega_{c}=5 \sim 10$ and approaches the polar values for larger $B_{o p} / \omega_{c}$.

As seen from fig.1, the carrier distribution is thin in the vicinity of the point $c$. This tendency becomes more pronounced with the increase of $\mathrm{B}_{\mathrm{op}} / \omega_{\mathrm{C}}$. Such a distribution corresponds to the population inversion over Landau levels, and a possibility of the NDC at $\omega_{c}$ has been pointed out $[6,7]$.

Superradiance and Linear Higher Harmonic Generation

Consider a distribution as Fig. 1 and assume that $\mathrm{E}_{\mathrm{x}}$ is suddenly removed at $t=0$. After $t=0$, the whole pattern of the distribution function begins to rotate with $\omega_{c}$ around the $p_{z}$-axis. This corresponds to a coherent circular motion of the carriers and brings about superradiance. Since the average induced dipole moment per carrier

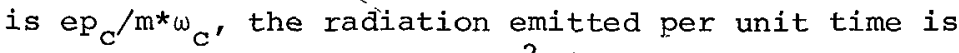

$$
\frac{d E}{d t}=-\frac{2}{3}\left(\frac{N e p_{C}}{m^{*} \omega_{c}}\right)^{2} \frac{\omega_{c}^{4}}{c^{3}},
$$

if $\mathrm{N}$ carriers rotate coherently. The radiation loss causes the damping of the coherent motion; the relaxation time ${ }^{T}{ }_{r}$ is given by

$$
\frac{1}{T_{r}}=\frac{4 \mathrm{Ne}^{2} \omega_{\mathrm{C}}^{2}}{3 \mathrm{~m}^{*} \mathrm{c}^{3}} \text {. }
$$

since the energy associated with the coherent motion is $\mathrm{Np}_{\mathrm{C}}^{2} / 2 \mathrm{~m}$. For instance, if we put $\mathrm{N}=10^{10}, \omega_{\mathrm{C}}=2 \times 10^{11} \mathrm{~s}^{-1}$ and $\mathrm{m}^{*}=0.1 \mathrm{~m}$, we get a very strong damping:

or

$$
\begin{aligned}
& \mathrm{T}_{r} \simeq 2 \times 10^{-11} \mathrm{~s}, \\
& \omega_{C^{T}} \simeq 4 .
\end{aligned}
$$

Namely, $\mathrm{T}_{\mathrm{r}}$ may be even shorter than the collision time $\tau$.

More generally, an appropriate rapid change in the electric field $\mathrm{E}_{\mathrm{X}}$ can also cause the similar effect. However, it is not a technically easy task to realize the rapid change of $E_{x}$ within a fraction of $\omega_{C}^{-1}$. A more feasible way is to apply an alternating field with a frequency $\omega=\omega_{c} / n$ ( $n$ is an integer) along the $x$-axis in addition to the static field $\mathrm{E}_{\mathrm{x}}$, although the resulting effect is a little different from the above one: The total electric field is

$$
E_{x}^{\prime}(t)=E_{x}+A \cos \omega t \text {, }
$$

and the free motion of a carrier is given by

$$
\begin{aligned}
& p_{X}(t)=-e A \frac{\omega}{\omega_{c}^{2}-\omega^{2}} \sin \omega t+a \sin \left(\omega_{c} t+\phi\right) \\
& p_{Y}(t)=e A \frac{\omega_{C}}{\omega_{c}^{2}-\omega^{2}} \cos \omega t-a \cos \left(\omega_{c} t+\phi\right)+p_{C}
\end{aligned}
$$

where a and $\phi$ are arbitrary constants. In this case, the carriers 
accumulate in a region as hatched in Fig.5. The region rotates with $\omega_{c}$ around the moving point $C^{\prime}$ that makes the elliptic motion (corresponding to that of $a=0$ in eq. (11)) around $c$ as shown in the figure. Because the point $C^{\prime}$ does not coincide with the center of mass of the accumulation region, a dipole moment rotating with $\omega_{c}$ is induced. If we assume the distribution to be uniform over the region, the average momentum on the $p_{z}=0$ plane is given by

$$
\bar{p}_{x}(t) \simeq \frac{e A}{\omega\left(n^{2}-1\right)}\left\{-\sin \omega t+\frac{2}{\pi} \frac{n^{2}}{n^{2}-1} \sin \frac{\pi}{n} \sin \omega_{c} t\right\}
$$

and $\quad \bar{p}_{Y}(t) \simeq \frac{e A}{\omega\left(n^{2}-1\right)}\left\{n \cos \omega t-\frac{2}{\pi} \frac{n^{2}}{n^{2}-1} \sin \frac{\pi}{n} \cos \omega_{c} t\right\}+p_{c}$ '

when $A$ is not too large. The

second term represents a peculiar

higher harmonic generation which is very strong and proportional to $A$, in contrast with the ordinary case proportional to $\mathrm{A}^{\mathrm{n}}$. The linear dependence comes from the approximation that the edge of the distribution is sharp. In reality, the edge has some small width $\Delta p$. If eA $/ \omega_{c}$ becomes smaller than $\Delta p$, the situation will approach the ordinary case.* Typically, the corresponding

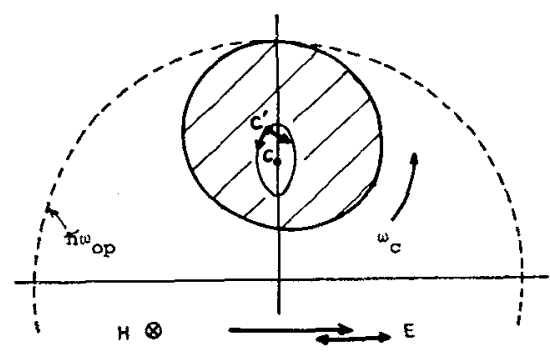

Fig.5. Carrier accumulation in static plus weak alternating (of $\omega / 2)$ fields. The arrow shows a rotating dipole moment which induces superradiance. value of $A$ is estimated to be $1 \sim 10 \mathrm{~V} / \mathrm{cm}$.

Even in the latter case, the radiation damping is still given by the same relaxation time as (9). Thus, if favourable conditions are prepared, one can expect to observe a sustained superradiance pumped by the field $E_{x}^{\prime}$ with appreciable damping that is occasionally comparable with the ordinary collision relaxation in crystals.

\section{p-Type semiconductors}

In the case of the warped band, the trajectory of a carrier in the momentum space under crossed electric and magnetic fields differs considerably from that in the spherical band. Furthermore in the case of p-type silicon, the band nonparabolicity has pronounced effects on the motion of holes. Recently, a negative Hall effect which presumably relates to that has been observed by Kajita [8] .

*There is a doubt about this, because of the singularity at the edge of the distribution function (for $A=0$ ) caused by the singular nature of the optical phonon emitting rate which is proportional to $\sqrt{\varepsilon-\hbar \omega_{o p}}$. 


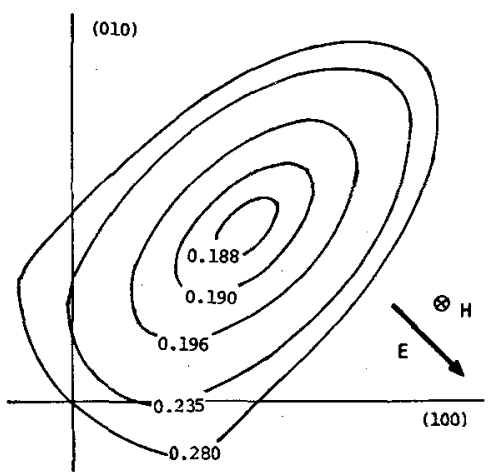

Fig.6. Trajectories of a heavy hole in Ge. The numbers denote the cyclotron mass in the unit of free electron mass.

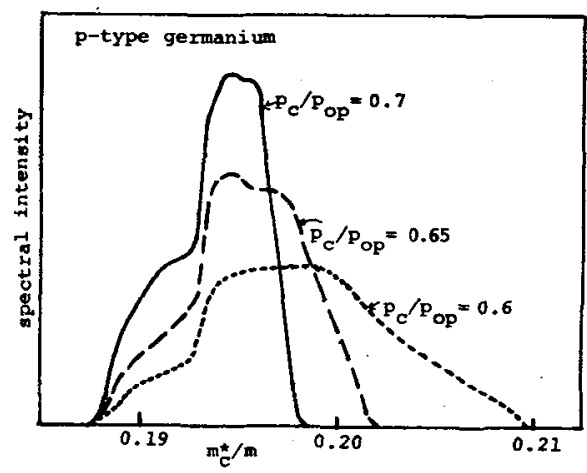

Fig.7. Spectral distribution of the cyclotron mass of the accumulated holes in $\mathrm{Ge}$.

An expected effect in p-type germanium associated with the band warping and the carrier accumulation is a specific shift of cyclotron resonance. Figure 6 shows the momentum space trajectories of a hole on the $\mathrm{p}_{z}=0$ plane under electric (1) germanium. Values of cyclotron mass along the trajectories are also shown in the unit of free electron mass. The band parameters are those given by Hensel and Suzuki [9]. The usual cyclotron mass on the $\mathrm{p}_{\mathrm{z}}=0$ plane is $0.28 \mathrm{~m}$. Thus, if the cyclotron resonance experiment is performed on the accumulated carriers, appreciable shift will be found. Fig.7 shows the expected spectral distribution of the cyclotron mass calculated by summing over all orbits (with appropriate weight) in the accumulation region, for three values of $p_{c} / p_{o p}$ ( $p_{o p}$ is the momentum at $\hbar \omega_{\text {op }}$ in (110) direction).

Another expected effect in p-type materials associated with the accumulation comes from the band degeneracy; the existence of light and heavy hole bands. If the ratio $\mathrm{E}_{\mathrm{X}} / \mathrm{H}_{\mathrm{z}}$ is suitable,

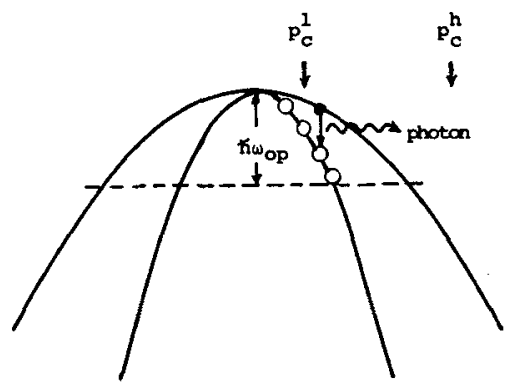

Fig.8. Accumulation in light hole band and induced photon emission. the accumulation takes place only in the light hole band and increases the portion of the light hole. In this case, population inversion between two bands and emission of infrared radiation (Fig.8) are expected [4]. 
\$3. Microwave Fields

If sufficiently strong microwave fields are applied, the carrier is repeatedly accelerated up to the optical phonon energy within a half cycle of the fields. We call this situation "streaming cyclotron motion", which is completely different from the usual cyclotron resonance condition. Some experimental evidences of such a motion have been found in AgBr [10]. A rapid increase of the line width and a pronounced peak shift with the field intensity have been observed (Figs.9 and 10). A Monte Carlo calculation has reproduced the experimental results very well and also supported the concept of the streaming cyclotron motion.

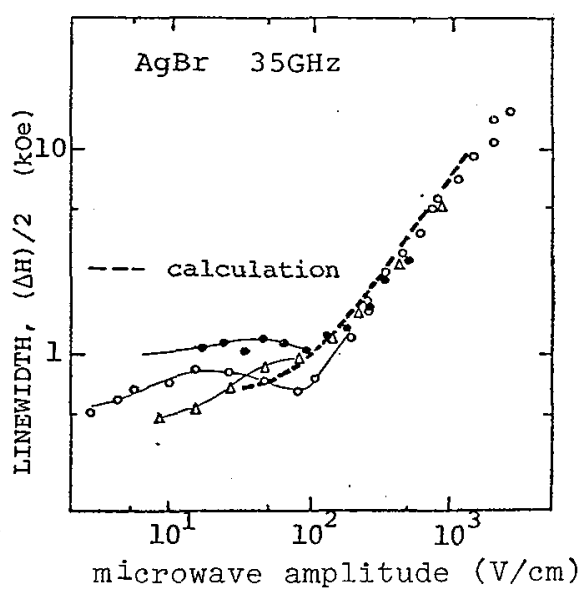

Fig.9. Half width of resonance line vs. microwave intensity.

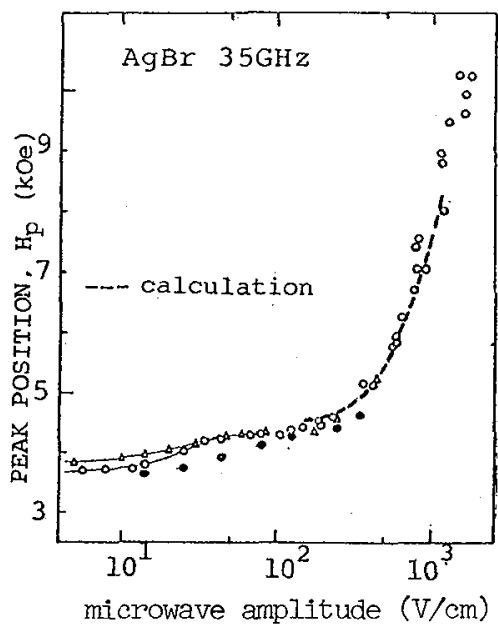

Fig.10. Variation in the peak position.

However, the details revealed by the calculation are even more interesting [11]. The motion of carriers is quite different from that expected from brutal acceleration by such intense fields but much more ordered. The distribution function and the shape of the response current exhibit peculiar features. Some of them are presented in Figs.11-16, which show the distribution functions on the $\mathrm{p}_{\mathrm{z}}=0$ plane. In the insets, the response current $J_{x}(t)$ (sometimes also $\left.J_{y}\right)$ to the field $E_{x}(t)=A \cos w t$ are plotted together with the indication of the corresponding time at which the distribution is recorded (the vertical arrows). All the calculations are carried out for $\mathrm{AgBr}$, in which the polar optical, acoustic and neutral impurity scattering processes are considered. The collision frequency by the impurity is fitted to give the experimentally observed low field mobility at $4.2 \mathrm{~K}$, $2 \times 10^{5} \mathrm{~cm}^{2} / \mathrm{V} \cdot \mathrm{s}$, and $\omega / 2 \pi$ is taken to be $35 \mathrm{GHz}$. In the case of $\mathrm{Fig} \cdot 1 \mathrm{l}$, $\mathrm{H}_{\mathrm{z}}=0$ and the carrier repeats the simple streaming motion between 


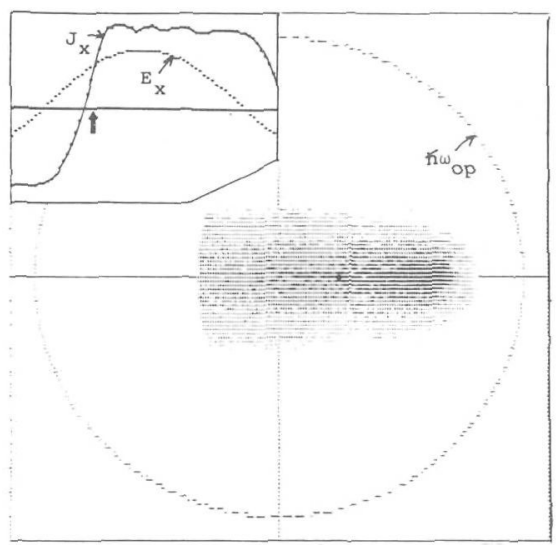

Fig. 11. $A=1200 \mathrm{~V} / \mathrm{cm}, \omega_{C}=0$.

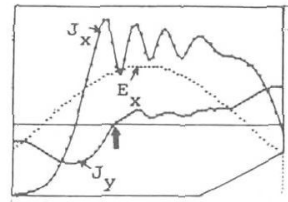

Fig.13. $A=1200 \mathrm{~V} / \mathrm{cm}, \omega_{C} / \omega=0.8$.

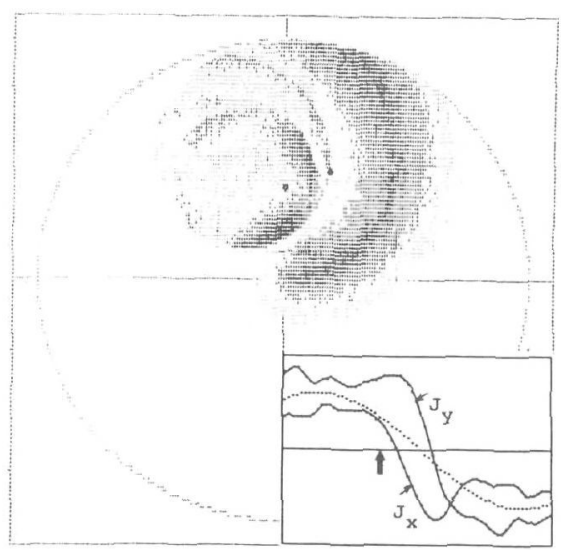

Fig.15. $A=1200 \mathrm{~V} / \mathrm{cm}, \omega_{C} / \omega=3.5$.

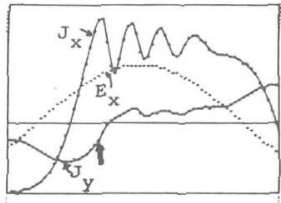

Fig. 12. $A=1200 \mathrm{~V} / \mathrm{cm}, \omega_{C} / \omega=0.8$.

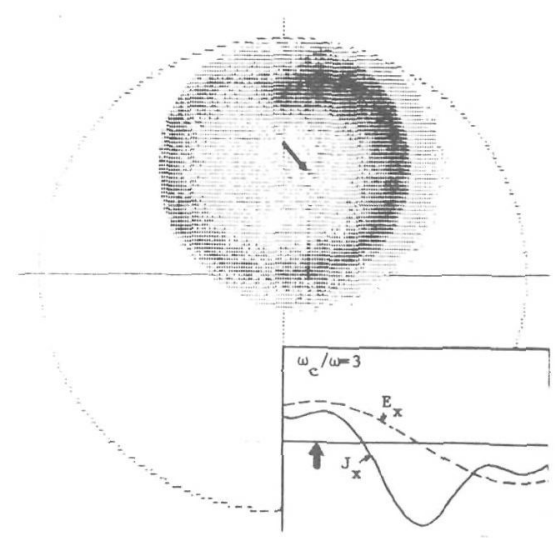

Fig. 14. $A=780 \mathrm{~V} / \mathrm{cm}, \omega_{C} / \omega=3$.

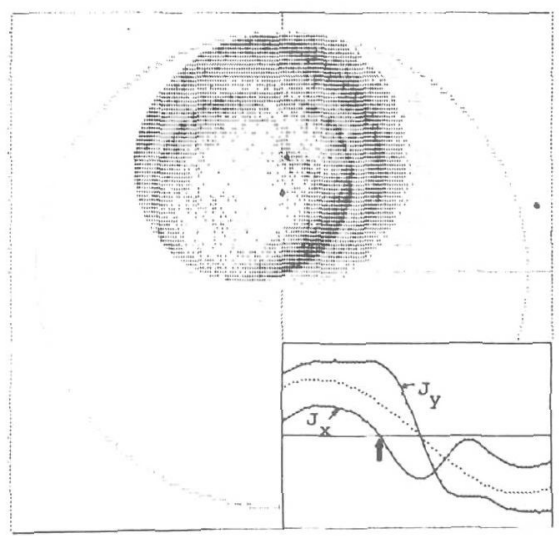

Fig. 16. $A=1200 \mathrm{~V} / \mathrm{cm}, \omega_{C} / \omega=4$. 
$p_{x}=-p_{o p}$ and $p_{o p} \cdot \quad$ Figures 12 and 13 are of two different times in the same series and show the bunching effect of the carriers in the p-space, the motion of the bunched carriers leads to sawtooth current [11]. The case of Fig.14 has some resemblance to that of Fig.5; the rotating dipole moment as indicated by the arrow induces the third harmonic generation. In the cases of Figs.15 and 16, the motion is rather disordered and the distribution exhibits complicated patterns, which are not the fluctuation due to the Monte Carlo calculation but reproducible.

\section{References}

1) H.Maeda and T.Kurosawa: Proc. IIth Int. Conf. Phys. Semiconductors, Warsaw 1972, p.602.

2) S.Komiyama, T.Masumi and K.Kajita: Phys. Rev. B20 (1979) 5192.

3) S.Komiyama and R.Spies: to be published in this conference.

4) A.A.Andronov, V.A.Valov, V.A.Kozlov and L.S.Mazov: Solid state Commun. 36 (1980) 603 .

5) T.Kurosawa, H.Maeda and H.Sugimoto: J. Phys. Soc. Jpn 36 (1974) 491.

6) Ya.I.Al'ber, A.A.Andronov, V.A.Valov, V.A.Kozlov and I.R.Ryazantseva: Solid State Commun. 19 (1976) 955.

7) T.Kurosawa: Solid state Commun. 24 (1977) 357.

8) K.Kajita: Solid State Commun. 31 (1979) 573.

9) J.C.Hensel and K.Suzuki: Phys.Rev. B9 (1974) 4219.

10) S.Komiyama, T.Masumi and T.Kurosawa: Proe. 14th Int. Conf. Phys. Semiconductors, Edinburgh 1978, p.335.

11) T.Kurosawa: Proc. 15th Int. Conf. Phys. Semiconductors, Kyoto $1980, p, 345$. 